\title{
Automated assembly of stacked sensor modules for the CMS outer tracker upgrade
}

\author{
James Keaveney* \\ Deutsches Elektronen-Synchrotron (DESY) Notkestraße 85, 22607 Hamburg, Germany \\ E-mail: james.keaveney@desy.de
}

\begin{abstract}
The inner part of the CMS phase II upgrade outer tracker will be built from $P S$ modules consisting of two closely spaces silicon sensors and associated electronics and mechanics. The sensors must be assembled with a translational (x-y) alignment of approximately $40 \mu \mathrm{m}$ and a rotational alignment of $800 \mu \mathrm{rad}$. An automated module assembly system is proposed as a method to build these modules on a large production scale. The automated system is based on the integration of a high-precision motion stage with vacuum handling and vision systems. A dedicated software application obtains images, performs pattern recognition to deduce component positions, and controls the motion and vacuum handling systems to precisely position components.
\end{abstract}

The 26th International Workshop on Vertex Detectors

10-15 September, 2017

Las Caldas, Asturias, Spain

\footnotetext{
*Speaker.
} 


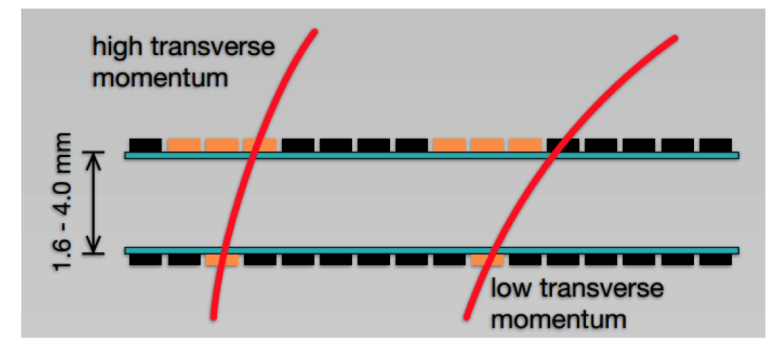

Figure 1: A schematic of the estimation of particle momentum in module volume is shown.

\section{Introduction}

The high multiplicity of particles produced in the collisions of the High-Luminosity LHC (HL-LHC) presents a significant challenge in the efficient recording of data by the CMS trigger system. In order to increase trigger performance, the CMS phase II upgrade outer tracker will provide tracking information from every bunch crossing to the first-level of the trigger. The transverse momentum $\left(p_{T}\right)$ of a charged particle is derived at the tracker-module level from consistency between the particle's trajectory as it traverses the module volume and the beamline in the transverse plane. In figure 1, a schematic of the estimation of particle momentum in module volume is shown. Pairs of hits in a given module passing a $p_{T}$ threshold (known as stubs) are read out by the trigger at the LHC bunch crossing rate. A track reconstruction based on the stubs is then performed allowing tracking-based criteria to be applied to select event for further analysis. A complete description of the CMS phase II tracker and the envisaged usage of tracking information in the CMS trigger is given in [1].

\section{The PS module}

The CMS phase II outer tracker will contain 5616 PS modules and $76802 S$ modules. The PS modules will populate the innermost region of the outer tracker and consist of one pixel sensor with dimensions $98.74 \times 49.16 \mathrm{~mm}$ and one strip sensor with dimensions $98.14 \times 49.16 \mathrm{~mm}$ which together produce the stubs crucial to the momentum reconstruction. An exploded view of a PS module is shown in Figure 2. The sensors are glued to a carbon-fibre reinforced Aluminium (Al$\mathrm{CF}$ ) spacers which maintain the distance between the sensors and provide the thermal conductance for cooling of the module. The two sensors and spacers are in turn glued the carbon-fibre (CF) baseplate. In order to reliably measure $p_{T}$, the sensors must align to within $40 \mu \mathrm{m}$ measured at the sensor's short edge. This corresponds to a rotational alignment tolerance of $800 \mu \mathrm{rad}$.

\section{Automated assembly}

In order to satisfy the precision alignment requirements of the PS module for a large scale module production campaign, an automated assembly procedure is proposed. In addition, the automated assembly mitigates the reliance on precisely diced sensors and clear access to the sensor edges associated with a manual, jig-based assembly. In this section, the basic hardware and software elements and fundamental capabilities of the system are described. 


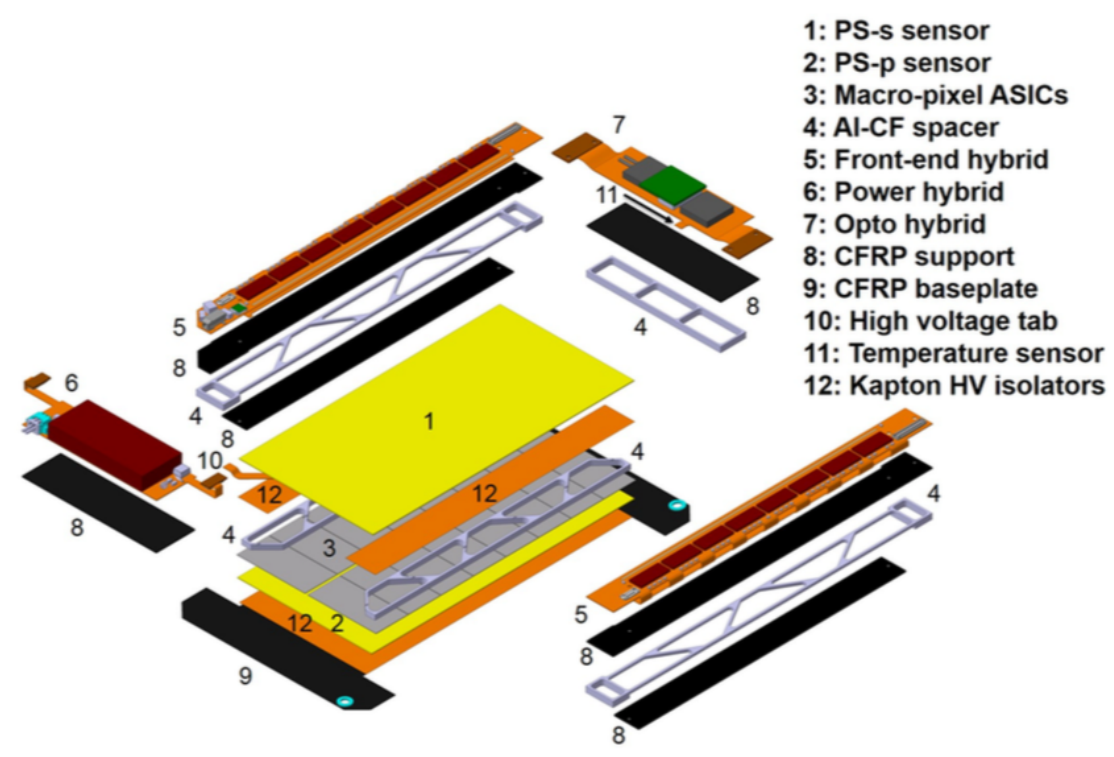

Figure 2: An expoded view of the PS module is shown with constituent parts indicated, taken from [1].

\subsection{Hardware}

The assembly setup consists of three sub-systems: the motion subsystem, the vision subsystem and the handling subsystem. The motion subsystem provides the precise mechanical movements needed to arrange the module components and contains two moveable parts: a $x-y-z$ stage that can move in $\mathrm{x}-\mathrm{y}-\mathrm{z}$ coordinates to a precision of $4 \mu \mathrm{m}$ and a stage that can rotate to a precision of $\approx 175 \mu \mathrm{rad}$. The motion stages are controlled by a controller unit which receives control signals and reports the status of the motion subsystem. All motion hardware is manufactured by Lang ${ }^{1}$. The handling system enables secure but non-destructive mounting of delicate module components to the motion stages. It consists primarily of a vacuum pump and two handling tools: the pickup tool and the assembly platform. Vacuum is distributed from the pump to the handling tools in two independent channels via plastic tubing. Switching of the channels is controlled by a fourchannel vacuum valve manifold that receives $12 \mathrm{~V}$ on/off signals from a relay card. The pickup tool consists of an ESD plastic block housing an inner vacuum chamber which distributes the vacuum to an array of downward facing ESD rubber suction cups on its lower surface. A custom-built Al tool known as the arm is mounted on the $\mathrm{x}-\mathrm{y}-\mathrm{z}$ stage allowing mounting of the pickup tool to the $x-y-z$ stage with adequate clearance the motion stage. The assembly platform is an Al block with precisely machined features for manual arrangement of the spacers and baseplate, it also houses two independent networks of vacuum inlets, outlets and connecting vacuum channels. These networks allow the spacers, sensor and baseplate to be vacuum-fixed. The assembly platform is mounted on the rotation stage. The vision system acquires images of the module components during the assembly procedure. When coupled with the pattern recognition routines described in the next section, this facilitates automatic calculation of the motions of the $x-y-z$ and rotation stages needed for alignment of the components. The vision system consists of a high-resolution, camera mounted

\footnotetext{
${ }^{1}$ Lang models: GT9-NSMA, LT-LBMA, RT5-NSMA
} 
on the arm in a downward-facing orientation. A image of the assembly setup is shown in figure 3.

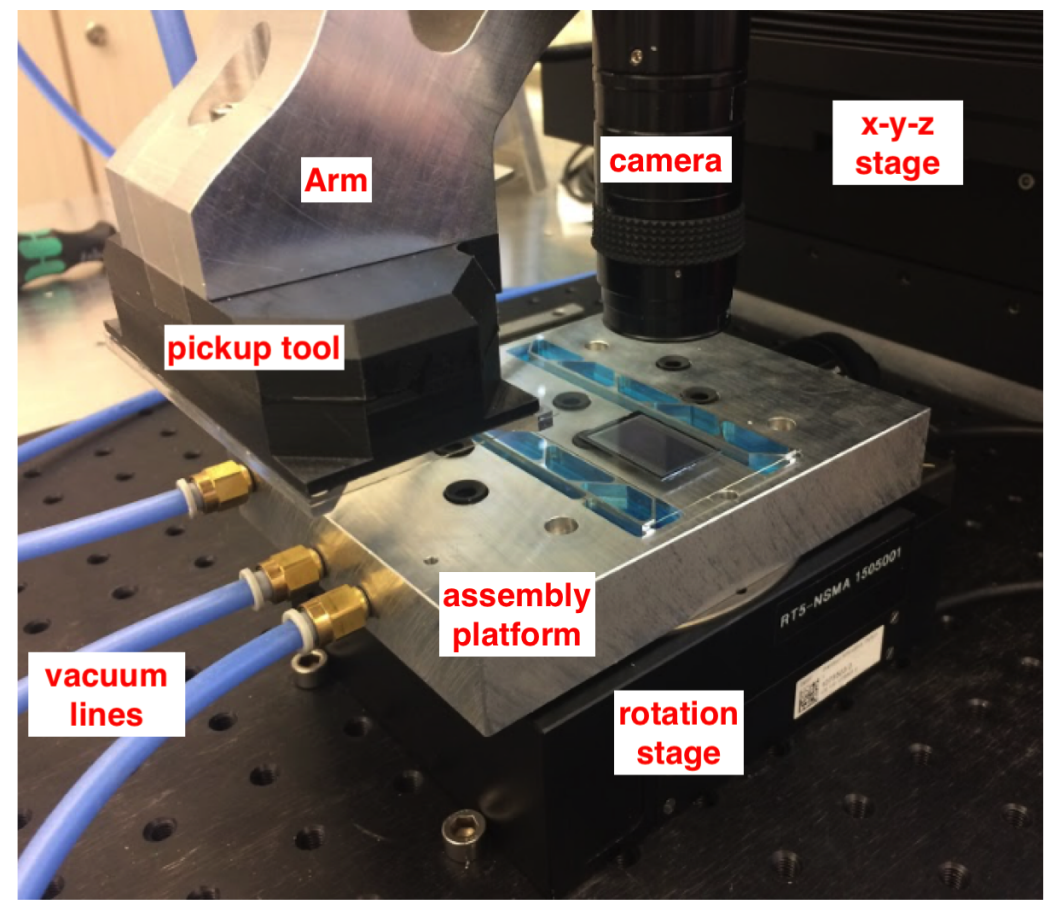

Figure 3: The hardware components of the assembly setup are shown.

\subsection{Software}

The motion, handling and vision systems are integrated via a dedicated software application. The application provides the control signals and monitoring needed to operate the motion system, acquire images and switch the vacuum channels. All data is transmitted via USB interfaces. User interaction occurs via a GUI written using the Qt framework version 4.8.7 [3].

\subsection{Fundamental capabilities of the system}

\subsubsection{Determination of position and planar orientation of sensors}

The determination of the location and planar orientation defined as the rotational orientation of the sensor in the horizontal ( $x-y)$ plane, of the sensors is now described. A dedicated pattern recognition algorithm analyses images of markers at each corner of the sensors. The markers are precisely positioned with respect to the strips or pixels or the sensors. Hence precise alignment of the markers ensures the alignment of the pixels or strips. The algorithm utilises the OpenCV [2] package to perform the fundamental manipulations and analyses of the raw images.

The raw images from the camera are first converted to binary images in which each pixel is either black or white in a process known as thresholding. Thresholding converts each pixel of the image into a white(black) pixel if the intensity of the pixel is above(below) a pre-defined threshold. Thresholding serves to reduce the differences between images due to noise arising from dust and random differences in the surfaces of the sensors. In addition, the differences in brightness of images causes by changes in ambient lighting can be suppressed by changing the threshold. The 


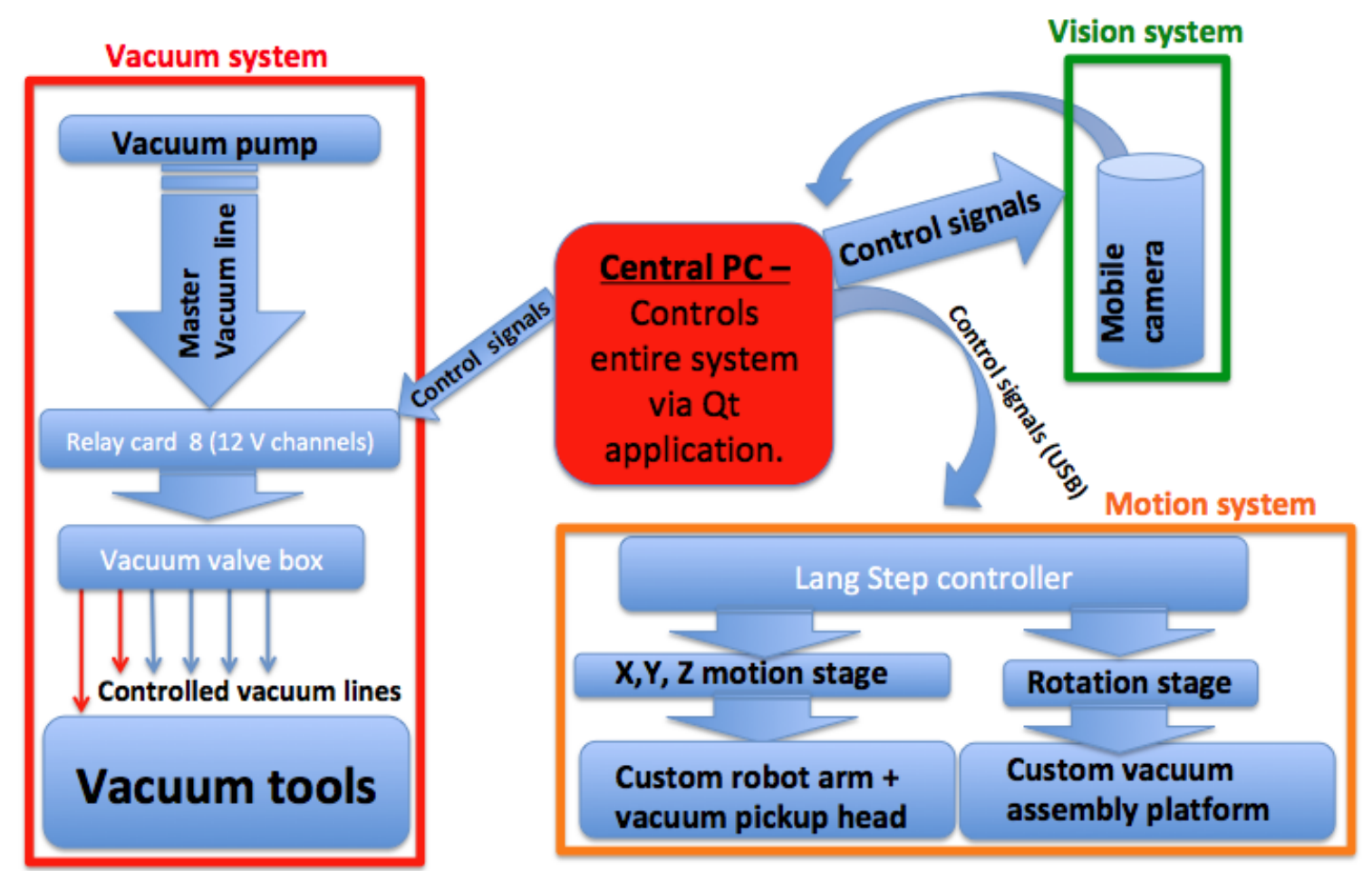

Figure 4: A schematic of the integration of the motion, vision and vacuum subsystems is shown.

$\mathrm{x}-\mathrm{y}$ position and planar orientation of the marker within the binary image is determined using a image processing technique known as template matching. In a template matching routine, a pattern pre-defined by a template image is sought within the master image under inspection. In this case, the master image corresponds to a binary image of the corner of the sensor currently being assembled and the template image corresponds to a binary image of a fiducial marker from a reference sensor. Template matching proceeds by superimposing the template image at each x-y point of the master image and calculating a metric which quantifies the similarity of the template image and the portion of the master image over which it overlaps. The point in the master image where the metric reaches a minimum represents the most probable location of the fiducial marker. In figure 5 the determination of marker position with template matching is illustrated. In order to make an initial estimate of the planar orientation of the marker, the procedure is repeated iteratively with different rotational transformations applied to the master image. The angle at which the minimal metric value obtained across all iterations is taken as an estimate of the planar orientation.

To make a more precise estimation of the planar orientation, the $x-y$ position of the diagonally opposite sensor marker is measured. The initial estimate of the planar orientation is used together with the $\mathrm{x}$ and $\mathrm{y}$ distances between the markers on the sensor to calculate the movement needed for the motion stage to move so that the camera has the diagonally opposite corner within its field of view. The location of the second corner is determined with the same procedure as was performed for the first corner. The two marker locations are then used to make a final determination of the $x-y$ location and planar orientation of the sensor. 

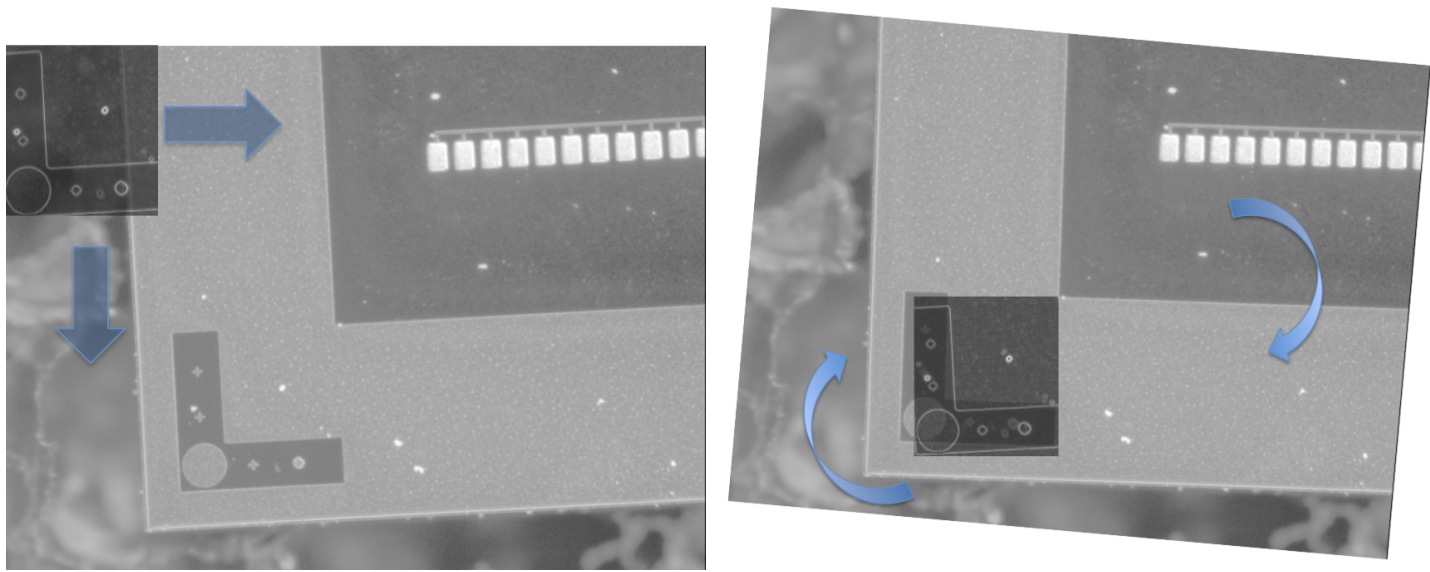

Figure 5: The technique of template matching is illustrated. In the left image, the blue arrows illustrate the scanning of the template across the master image. In the right image, the blue arrows illustrate the rotations applied to the master image to deduce the rotational orientation of the sensor with respect to the template image.

\subsubsection{Pickup and set down}

The pickup refers to the procedure whereby a sensor resting on the assembly platform, referred to here as the top sensor, is transferred to the pickup tool. The set down refers to the procedure whereby the top sensor is transferred to a pre-determined $x-y$ location on the assembly platform. With the pickup tool centered over the top sensor, the motion stage lowers the pickup tool until the suction cups of the pickup tool are in contact with the upper surface of the top sensor. The vacuum of the pickup tool is then engaged and the top sensor attaches to the pickup tool. The $x-y-z$ stage can now move upwards to move the top sensor. In the set down procedure, the aim is to detach the top sensor from the pickup tool at a x-y location to exactly coincide with the corner marker of the bottom sensor placed on the assembly platform. In order to position the top sensor so its $x-y$ location and orientation matches that of the bottom sensor, one first measures the $x-y$ location the bottom sensor. The required motion is then applied to the $x-y-z$ stage.

\subsubsection{Fast gluing}

Module components are joined using glue. To satisfy the heat conduction and radiation hardness requirements of the module in the operational environment of the CMS tracker, the envisaged glue for adhesion of the sensors, spacers and baseplate is Polytec 601. However, Polytec 601 has a curing time of $\approx 24$ hours. This severely limits the throughput of the automated assembly system. Thus we supplement the Polytec 601 glue with an additional fast glue at the corners of the spacers and sensors corresponding to total surface area of $2-3 \mathrm{~mm}^{2}$ that will provide sufficient adhesion to allow movement of the assembly while preserving the alignment after a curing time of approximately ten minutes. The current choice for fast glue is Loxeal 31-42.

\section{First protoypes}

In order to commission the basic functionalities of the system and test the assembly proce- 
dures, prototype assemblies consisting of two dummy glass sensors and two dummy Al spacers. The dimension of the dummy components match very closely the dimensions of the nominal components. In lieu of fiducial markers at the sensor corners, scrap silicon structures with suitable fiducial markings are glued to the dummies to facilitate commissioning of the pattern recognition procedure. In figure 6 a photo of one of the prototypes is shown. The alignments of the two silicon structures is measured using a microscope and is found to be approaching the required alignment precision. Improvements in precision are expected via optimisation of the handling methods.

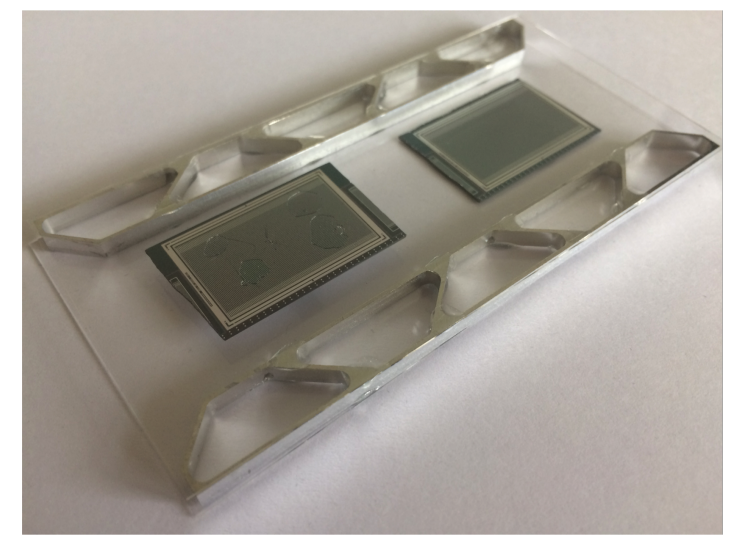

Figure 6: A prototype assembly is shown. The assembly consists of two dummy glass sensors with scrap silicon structures to provide fiducial markers and two dummy Al spacers.

\section{Conclusion}

An automated setup designed to assemble PS modules for the CMS phase II outer tracker upgrade is presented. The setup is based on motion, vision and handling systems integrated via a dedicated software application. First prototypes have been produced to demonstrate the basic functionalities of the system. As the project is in a commissioning phase, improvements in precision are expected, especially from optimisation of the handling methods.

\section{References}

[1] K. Klein, CERN-LHCC-2017-009, CMS-TDR-014.

[2] https://opencv.org

[3] https://www.qt.io 